811.131.1:811.163.41

https://doi.org/10.18485/italbg.2021.1.3

Mirela Boloban*

Università di Sarajevo

\title{
IRONIA E INTERVENTI DEL NARRATORE NELLE TRADUZIONI DEL PRIMO CANTO DELL'ORLANDO FURIOSO IN SERBO(CROATO)
}

\begin{abstract}
Nel presente contributo ci proponiamo di analizzare alcuni elementi delle traduzioni del primo canto dell'Orlando furioso, nel tentativo di comparare i risultati ottenuti dai diversi principi traduttivi utilizzati da Dragiša Stanojević e Danko Angjelinović. I due traduttori hanno propeso per differenti priorità e norme traduttive e di conseguenza, gli interventi del narratore, l'ironia, le parole e le espressioni ambigue legate allo specifico contesto storicoculturale ariostesco appaiono in maniera significativamente diversa nei due metatesti. Obiettivo finale della nostra analisi è mostrare, mediante il metodo comparatistico, i risultati dell'impiego dei differenti principi traduttivi che hanno creato un Orlando adattato al cronotopo serbo e un altro, vero ambasciatore della cultura e letteratura italiana nel territorio ex-jugoslavo, entrambi legati alle circostanze storicoculturali dei traduttori e alle aspirazioni delle epoche in cui le traduzioni sono state eseguite.

Parole chiave: principi traduttivi, interventi, cronotopo, corte, ironia, ambiguità.
\end{abstract}

\section{INTRODUZIONE}

L'Orlando furioso di Ludovico Ariosto non è solo il capolavoro del Rinascimento italiano, ma è anche uno dei più originali poemi cavallereschi mai scritti. Il valore del Furioso è stato riconosciuto anche nel territorio dell'ex-Jugoslavia, sebbene l'idea di tradurlo sia stata una grande sfida che pochi hanno osato affrontare, a causa della lunghezza e della complessità nel rendere lo spirito e il linguaggio ariosteschi. Ad oggi, esistono due traduzioni del Furioso in questo territorio: quella in serbocroato di Dragiša Stanojević Bijesni Rolando (1895) e quella croata di Danko Angjelinović Bijesni Orlando (1953). Data la distanza temporale e la posizione geografica che separa le traduzioni, è indubbio che i due traduttori appartengano a contesti culturali e storici molto differenti fra loro e che l'idea di comparare

*mirela.boloban@ff.unsa.ba 
e misurare i risultati delle loro traduzioni possa quindi sembrare azzardata. Comunque, tenendo conto che si tratta delle uniche due traduzioni del poema nelle lingue di questi due Paesi vicini e che le loro traduzioni sono così diverse, l'idea di analizzarle ci è sembrata molto opportuna per comparare i due diversi modi di interpretare l'opera ariostesca, l'impiego di due opposti principi traduttivi rispetto al prototesto, e di conseguenza i due diversi modi di ricezione. Le differenze inconciliabili fra le traduzioni, analizzate attraverso un confronto comparatistico, serviranno anche a mostrare la ricchezza e la complessità del poema originale e le varie possibilità interpretative del verso ariostesco, scoprendone l'ambiguità e le sfumature espressive.

Siccome l'originalità e l'innovazione che L. Ariosto porta nel genere epico-cavalleresco si fondano particolarmente su un'ironia complessa che si rispecchia nell'uso delle tecniche narrative, il modo in cui tutti questi elementi vengono trasmessi al lettore di un contesto non italiano sono una grande sfida e una dimostrazione cruciale delle qualità del traduttore. Com'è evidente già nelle traduzioni del titolo, si può discutere sulle differenze piuttosto che sulle somiglianze fra i lavori. La loro comprensione o l'analisi del testo, come primo passo nel processo di traduzione, le loro scelte e le priorità diverse nella traduzione/trasmissione sono condizionate dal contesto culturale, dalla posizione geografica dei loro Paesi rispetto all'Italia e dalla distanza temporale tra le traduzioni. Come mostreremo, i metatesti sono diretti particolarmente da due principi diversi nella traduzione: D. Stanojević ha propeso per l'accettabilità, mentre D. Angjelinović ha scelto di servirsi del principio dell'adeguatezza, non riscontrando ostacoli culturali e linguistici nell'ambiente e nel pubblico designato.

\section{L'ATTO DEL TRADURRE}

Secondo Steiner (1998: 297), "il traduttore invade, estrae e porta a casa". Tradurre bene significa trovare un compromesso (Levy 1982: 58); non è possibile trasmettere il prototesto senza deviazioni e mancanze nel senso o nello stile. Il procedimento di trasmissione di qualsiasi opera straniera comprende un lavoro faticoso che diventa ancora più esigente nel caso della poesia. Nella prima fase del processo traduttivo, l'analisi, il vantaggio e il fattore più significativo è l'esperienza biculturale, un aiuto iniziale che però, durante la sintesi, può essere assolutamente perso se il traduttore non mostra una vera abilità e sensibilità nella valutazione delle capacità e delle aspettative del pubblico. Secondo Morini (2015), per ottenere una buona traduzione, il traduttore deve essere assolutamente biculturale, sebbene questa condizione non sia la garanzia di una traduzione riuscita: Levy (1982: 13) sottolinea che il traduttore, accanto alla buona conoscenza della 
letteratura e dei realia del Paese in cui si parla la lingua dalla quale traduce, deve tenere ugualmente in conto i propri lettori e la loro tradizione letteraria: "Se non trova un legame con i suoi lettori nella lingua materna, molte qualità del suo lavoro saranno incompresi e la sua traduzione sarà futile e fallimentare". Esiste un'opposizione delle due norme nel processo dello sviluppo dell'arte riproduttiva: della "norma della riproduzione" e della "norma artistica" (70). La prima norma si basa sul criterio della fedeltà e del senso, mentre la seconda si concentra sulla bellezza. La fedeltà o "la verità artistica" (71) è stata cronologicamente opposta alla bellezza, dipendendo dell'epoca. Secondo J. Levy, per raggiungere una traduzione buona, riuscita, il traduttore non si deve limitare a solo una delle norme, ma è necessario impiegarle entrambe in una stessa traduzione. Osservando i risultati di una traduzione, dopo la ricerca delle fonti dell'autore, si passa all'analisi "del metodo traduttivo" e della "concezione traduttiva" (216). J. Levy sottolinea che ogni valutazione di una traduzione comincia con un'attenta analisi e una comparazione con l'originale, alla ricerca di deviazioni. Queste deviazioni sono sempre "fatti delle culture di destinazione" (Toury 2012: 23) e possono caraterizzare "il rapporto dello stile dell'epoca e dello stile individuale del traduttore riguardo allo stile dell'autore, e anche l'approccio traduttivo rispetto all'idea oggettiva dell'opera." (Levy 1982: 217).

Occasionalmente, il traduttore deve per forza sacrificare una parte del senso o dello stile del prototesto per non perdere l'attenzione e la comprensione dei lettori. Comunque, la vera padronanza sul testo e le abilità del traduttore si osservano nella misura e nelle "compensazioni" (Levy 1982: 131) nella traduzione, evitando di sfociare in una libertà eccessiva nell'adattamento. U. Eco (2013) rileva che in molti casi il traduttore si lascia portare troppo lontano dal proprio contesto, tenendo solo in conto i suoi destinatari e a causa di troppa libertà, il risultato non è la creazione di una nuova opera; allontanandosi troppo dal prototesto, il traduttore ottiene una nuova versione, non una traduzione. Questa debolezza della traduzione può essere la conseguenza del principio traduttivo chiamato accettabilità, dove per definizione domina la cultura ricevente (Toury 2012). Dall'altro lato, il principio dell'adeguatezza, dove dominano elementi dal cronotopo del prototesto (Toury 2012), se i realia delle due culture (della cultura del prototesto e del metatesto) sono troppo lontane, può portare a un'incomprensione e a un disinteresse da parte dei lettori.

U. Eco (2013: 129) avverte che:

Ci sono delle traduzioni che arricchiscono splendidamente la lingua di destinazione e che, in casi che molti ritengono fortunati, riescono a dire di più (ovvero, sono più ricche di suggestioni) degli originali [...]. Una traduzione che arriva a "dire di più" potrà essere un'opera eccellente in sé stessa, ma non è una buona traduzione. 
Questa citazione descrive esattamente, come vedremo, la scelta del principio tradutttivo e i risultati ottenuti dalla traduzione Bijesni Rolando di D. Stanojević.

\section{BIJESNI ROLANDO}

D. Stanojević, poeta, traduttore e politico, traduce il Furioso e lo fa pubblicare nel 1895 a Belgrado, dalla Cooperativa letteraria serba. Le reazioni della critica riguardo alla traduzione erano numerose, prevalentemente negative e incentrate sulla questione dell' eccessiva libertà, anche per l'epoca in cui svolse il lavoro: S. Musić (1975: 594) scrive che non serve a niente mostrare le scorrettezze e l'arbitrarietà nella versione di Stanojević dell'Orlando furioso "perché ciò non ci porterebbe da nessuna parte" e che il traduttore è diretto da "un ardente desiderio d'istruire". . Marko Car esprime un giudizio ancora più severo e critico nel commento a D. Stanojević: "ogni analisi più seria indica che ci sono grandissime rotture della struttura nella traduzione di D. Stanojević, tanto che nel serbocroato è apparso un poema epico [sic] che non è né elegante, né armonioso né rinascimentale, ma "un poema ironico-comico" (Musić 1975: 595).

L'intento di D. Stanojević era chiaro ed espresso in forma di lettere, scritte alla Cooperativa letteraria serba dal traduttore stesso. Stanojević ci espone il suo punto di vista sulla traduzione eseguita e sulla situazione della letteratura serba in generale. Da queste lettere si può capire che la sua decisione di tradurre questo testo non ha origine tanto nel desiderio di avvicinare L. Ariosto ai lettori del suo paese, quanto piuttosto nel bisogno di avvalersi del modello ariostesco per rinnovare la poesia serba e sfoggiare la sua abilità di riscrivere un poema cavalleresco italiano, ma con un timbro serbo.

Il Rolando presentato ai serbi dalla Cooperativa, non è quello che Ariosto ha regalato agli Italiani, perché quello lì non sarebbe stato capito dai serbi. Questo Orlando è una mia invenzione: è un bambino a cui Ariosto ha dato l'anima e a cui io ho dato ossa e carne serbe (Stanojević 1895: 396).

Le lettere che spiegano il componimento del poema, le difficoltà, gli ostacoli riscontrati nel procedimento, nonché le intenzioni e gli scopi letterari di D. Stanojević per il futuro della poesia serba sono poi state pubblicate come prologo al poema. Si tratta di scritti che servono come chiave di lettura e di analisi del poema. Gli scopi letterari e la norma adottata da D. Stanojević corrispondono alla prevalenza della norma artistica nel romanticismo, che tende a "stabilire le caratteristiche nazionali e individuali" tramite la riproduzione artistica. (Levy 1982: 71) Con quest'idea D. Stanojević esegue

\footnotetext{
${ }^{1}$ Tutte le traduzioni in questo lavoro sono eseguite dall'autrice del contributo.
} 
anche le traduzioni di altri capolavori italiani (della Divina Commedia e della Gerusalemme liberata) precisando nell'epilogo del Bijesni Rolando che l'obiettivo finale è offrire un contributo soprattutto patriottico, conformemente alla visione che le traduzioni "sono l'intermediario che dirige lo sviluppo letterario nella direzione della letteratura mondiale" (Levy 1982: 229). Anche la conclusione del contributo di M. Zogović ricorda che il traduttore principalmente tenta di avvicinare le opere più grandi al suo popolo, definito da egli stesso come "grandeserbo" basandosi sulla seconda lettera scritta alla Cooperativa: "traducendo [gli autori] più geniali e, per la tecnica del verso, i più difficili da tradurre, $i$ classici moderni $[\ldots]$ mi propongo di aprire la strada alla nostra lingua in tutto il sud-est sloveno [...] diffondendo il pensiero che nei Balcani vi sia posto solo per una lingua letteraria slovena, quella serba" (Zogović 1972: 390). L'accettabilità come principio è una scelta logica anche per i seguenti motivi: D. Stanojević non percepisce completamente l'ambiente e il linguaggio cortigiano del Rinascimento italiano, non ha i vantaggi del contesto di D. Angjelinović. S. Musić (1975: 601) sottolinea il fatto che il traduttore si sia servito maggiormemnte delle traduzioni tedesche del Furioso, rispetto all'originale, "il che senza dubbio riduce il valore della sua opera". Questa osservazione corrisponde al pensiero di Levy (1982: 216), concernente la valutazione del contributo creativo di un traduttore: "se il traduttore si appoggia più su un'altra traduzione che sull'originale, le differenze tra l'originale e la sua traduzione saranno di conseguenza maggiori”.

\section{BIJESNI ORLANDO}

La traduzione del Furioso eseguita da $\mathrm{D}$. Angjelinović è pubblicata da ZORA, a Zagabria, nel 1953. Il contesto croato è stato molto favorevole alla pubblicazione di un capolavoro italiano in lingua croata, siccome non mancavano lettori che già conoscevano bene la letteratura italiana e anche quella ariostesca. L. Ariosto era già noto nel territorio croato del Cinquecento, e ha suscitato un enorme interesse dato dai forti legami linguistici, culturali e storici fra la Croazia e l'Italia ${ }^{2}$. Lj. Avirović (2006: 83) proclama che "già agli albori della letteratura rinascimentale croata, a Split/Spalato e a Dubrovnik/Ragusa, per passare poi su tutto il territorio della costa [...] e del continente croato [...], i modelli e la traduzione di Dante Alighieri, di Francesco Petrarca e di Torquato Tasso erano diventati l'attività primaria per costruire le fondamenta del verso e talvolta per arricchire la letteratura locale in genere".

${ }^{2}$ Per avere un'idea generale sul legame e sull'influsso della letteratura italiana nel Rinascimento croato, abbiamo consultato Kadić (1962) “The Croatian Renaissance". 
Comunque, il Furioso dovrà aspettare il Novecento per essere presentato in croato: solo D. Angjelinović osa affrontare la complessità dell'opera, particolarmente interessato a offrire questo contributo ai lettori non italofoni dell'epoca. Traducendo il Furioso, arricchisce la conoscenza croata sulla letteratura rinascimentale italiana, presentando l'opera nella sua lingua materna, sempre mantenendo i riferimenti culturali ariosteschi. In tale modo, i suoi lettori, i quali sono già stati in contatto con la civiltà italiana da tante generazioni, hanno uno sguardo verosimile nel mondo rinascimentale creato di L. Ariosto.

Questo poeta e traduttore di Macarsca, dopo aver passato la Seconda guerra mondiale imprigionato in Italia, torna a Zagabria dove finalmente pubblica la sua traduzione. Avendo l'occasione di conoscere direttamente la cultura italiana, il suo vantaggio, rispetto a $\mathrm{D}$. Stanojević, si trova già nella prima fase preparativa di una traduzione: lui percepisce e comprende meglio l'ambiente e le particolarità del linguaggio del prototesto. L'altro vantaggio, che dirige la scelta verso il principio dell'adeguatezza è, accanto all'esperienza personale e la comprensione, la posizione geografica e le vicende storico-culturali comuni ai due Paesi: non solo il traduttore, ma anche la maggioranza dei lettori era in grado di capire l'ambiente italiano per via della "plurisecolare presenza della lingua italiana" particolarmente "nell' area dalmata costiera, nel Quarnaro e nella penisola istriana" (Malinar 2002: 284). In questo caso, si può vedere che il traduttore e i lettori sono biculturali e le circostanze sono state proficue per ottenere una traduzione accettabile anche senza molti adattamenti relativi all'appartenenza geografica e culturale del traduttore. Conseguentemente, a differenza del Rolando, il suo Orlando sembra avere tratti caratterizzanti molto più vicini a quelli di L. Ariosto e il linguaggio e l'ambiente immaginato nella traduzione corrispondono maggiormente all'immagine ideale di civiltà italiana rinascimentale. Lo spirito croato e il contesto culturale di Angjelinović non vengono nemmeno incorporati implicitamente nel poema: si potrebbe dire che si tratti di una traduzione, se non sempre fedele, quantomeno "neutrale", il che è sempre una buona scelta (Levy 1982). D. Angjelinović evita l'arbitrarietà, cerca equivalenti plausibili sostituendo ciò che trova intraducibile, non inventa, ma nemmeno banalizza l'originale. Ciononostante, mostreremo esempi dove la finezza dell'espressione, l'ironia complessa ${ }^{3}$ e l'ambiguità dell'espressione ariostesca mancano, come debolezze della traduzione rispetto all'originale.

${ }^{3}$ Sulla complessità dell'ironia nell'opera, scrive Rivoletti (2014: 14): 'L'ironia del Furioso è complessa e multiforme, come rivela anche solo l'analisi di poco più di una decina di ottave: è un'ironia che si dispiega a vari livelli del testo, dai commenti del narratore, alla disposizione e all'intreccio (per dirla con Hegel: alle "casuali" «collisioni») 


\section{GLI INTERVENTI DEL NARRATORE E L'IRONIA ARIOSTESCA}

Nelle seguenti parti del contributo, prendiamo in considerazione gli interventi come parte importante della realtà immaginata del poeta, comparandoli con i modi in cui sono stati tradotti/adattati nei metatesti. L'ambito culturospecifico del Furioso è profondamente legato alla corte rinascimentale e alla tradizione letteraria italiana. Le inclinazioni di un autore dell'ambiente cortigiano sono sempre presenti nella sua opera e scaturiscono in un rapporto ambiguo nei confronti della corte. Il modo in cui si rivolge ai suoi lettori corrisponde a certe regole legate all'epoca e alla tradizione dei poemi cavallereschi: l'uso del linguaggio formale e aulico ariostesco ha fini ironici.

S. Jossa (2017: 188) nel Lessico critico dell'Orlando furioso sottolinea il legame tra gli interventi del narratore e l'ironia, commentando un verso famoso del Furioso: "Socrate è del resto il nume tutelare di un'ironia cortigiana, tra Pontano e Castiglione, che si esprime soprattutto in una parola capace di spiazzare l'interlocutore e smascherarne le contraddizioni, che si riverbera dalla parola del narratore che commenta (Forse era ver, ma non però credibile)".

Gli interventi del narratore, che servono a "intensificare o pausare il ritmo del racconto all'interno di categorie che riassumono l'esperienza emotiva di quel pubblico e di quel genere letterario" (Praloran 1990: 109), contribuiscono enormemente alla lettura ironica dell'Orlando ariostesco. Le osservazioni generali del narratore, che interrompe bruscamente una scena commovente o una battaglia nel suo culmine, provocano l'effetto auspicato da Ariosto: il lettore si distacca dal passo e scruta quanto appena letto con uno sguardo più critico e riflessivo. Contemporaneamente, questa "pausa" rende la lettura ancora più divertente e ironica, grazie alla "sviluppo di uno spazio patetico" (Praloran 1990: 109) e funge altresì da passaggio, ci muove in un'altra scena in cui possiamo incontrare personaggi nuovi oppure quelli che abbiamo lasciato in precedenza.

Nella decima strofa, l'intervento del narratore che produce l'abbassamento, un altro procedimento vicino allo straniamento e amato da Ariosto, appare mediante l'uso di un paragone per avvolgere il personaggio in luce ironica:

Indossò la corazza, l'elmo in testa,

a spada al fianco, e in braccio avea lo scudo;

e più legger correa per la foresta,

ch'al pallio rosso il villan mezzo ignudo. [...]

(Ariosto 2016: 38, 10)

delle storie dei personaggi, allo scelto e sapiente eloquio (nel quale andranno compresi, come abbiamo visto, anche i giochi rimici)". 
Il momento di tensione, quando Angelica s'accorge di Rinaldo che si avvicina a lei, è interrotto dal paragone con il villano. Comparare Rinaldo, un personaggio eroico, a un semplice contadino, ha come conseguenza la rivelazione della natura umana dell'eroe e la completa distruzione dell'immagine quasi sovrumana di un paladino, che appare tradizionalmente nel genere epico-cavalleresco e finalmente, quindi, una lettura ironica. D. Stanojević perde l'ironia di questi versi e non traduce questo paragone. Nella sua versione Rinaldo trčao je brzo bez stanka $i$ mira "correva senza sosta e serenità" (Stanojević 1895: 3: 10).

Senza le tecniche narrative come l'entrelacement che "consente una maggiore varietà delle avventure e anche qualche effetto riuscito di suspense" (Praloran 1990: 79) e la pluralità di prospettive nella narrazione, il Furioso probabilmente non avrebbe avuto come conseguenza una lettura ironica. L. Ariosto tiene alla varietà dei personaggi e dell'azione e alle particolarità dei punti di vista, non solo come strumento di ironia, ma anche di auto-ironia: "adottare volta per volta un'infinità di punti di vista, di modo che si correggano reciprocamente; sfuggiamo a tutti i centrismi unilaterali, ritroviamo l'imparzialità e la giustizia della ragione" (Jankelevitch 1997: 39).

Nei versi della seconda strofa, quando L. Ariosto s'immedesima a Orlando che per amore venne in furore e matto e chiede Alessandra Benucci di lasciargli il poco ingegno che gli [...] basti a finir quanto ha promesso (Ariosto 2016: 35-36, 2) si perde la distanza tra l'autore, il personaggio, e l'opera stessa, dove appare 1'auto-ironia di L. Ariosto: "tra finzione e realtà si apre un dialogo" (Jossa 2017: 189). Come risultato, si crea l'effetto dell' $i$ ronia romantica: apparendo in prima persona come partecipante diretto di discussioni o dialoghi, l'autore si immedesima nella sua creazione, rompe il muro che si imponeva tradizionalmente tra la finzione e la realtà, separando l'autore o l'artista della sua creazione e del suo pubblico (Muecke 1986). D. Stanojević preserva il legame diretto tra Orlando e il suo autore traducendo il paragone tra i due: se da colei che tal quasi $m$ 'ha fatto (Ariosto 2016: 36, 2) in te s'i moj duh blizu ludila sroza "anche il mio spirito portò sull'orlo della follia", mentre D. Angjelinović non trasmette il paragone diretto tra i due e scrive soltanto: dopusti ako ona Uzorita / śto malo uma kap mi po kap pije "se mi permette la sua eminenza / che mi beve il sangue, goccia a goccia" (Angjelinović 1953: 7, 2).

Nonostante il precedente esempio di traduzione fallita di una scena ironica di D. Angjelinović, nella traduzione di D. Stanojević sembra che gli interventi siano trattati molto più spesso con superficialità, oppure che ve ne siano dove L. Ariosto non li ha messi. Così, succede frequentemente che la presenza del narratore non venga rispettata. Lo scrivere in prima persona è molto frequente in D. Stanojević, mentre D. Angjelinović evita di farlo, restando fedele a L. Ariosto. La forma impersonale dei versi (Ariosto 2016: 
46, 40): Cominciar quivi una crudel battaglia viene tradotta da Angjelinović (1953: 8, 40) come: I ljuta borba među njima poče "E una feroce battaglia fra di loro cominciò" mentre D. Stanojević spesso riporta la presenza del narratore come testimone dell'avvenimento (1895: 4, 40): Neću duljit opis ove ljute bitke "Non parlerò a lungo di questa feroce battaglia". Lo stesso succede nei versi: altra ventura al buon Rinaldo accade, che da costui tenea diverse strade (44: 31) come $O$ Rinaldu, koji drugim putem onim udari, sad ću malko da zazvonim "di Rinaldo, che prese l'altra strada, adesso dirò qualcosa" (7:31).

Come precedentemente illustrato, non è solo arbitraria la presenza del narratore in D. Stanojević. Stanojević è lontano dal rispettare anche altri aspetti formali del verso e le tecniche narrative. Gli enjambements "come strumento di tensione psicologica [...] pur nella dimensione narrativa, che come strumento di dinamismo cinetico [...]" (Praloran 2009: 179) non lo preoccupano, e spesso il senso degli ultimi due versi di una strofa viene incorporato nella strofa successiva. D. Angjelinović cerca di rispettare gli enjambements, così come i chiasmi nel poema, e lo fa con molto successo. Ad esempio, traduce: tanto gaudio o stupor tanto come s toliko čuda i sreće velike, preservando il chiasmo (1953: 11, 53), mentre D. Stanojević lo semplifica in: začudi tako $(1895: 11,53)$. Tenendo conto soltanto della rima, D. Stanojević non esita ad aggiungere dei climax completamente inventati. Nella strofa quarantasette inserisce queste parole e rende la scena molto più drammatica di quella originale: cvili, pišti, bradu, kosu kida "geme, strilla, strappa la barba e i capelli" $(1895: 10,47)$. Tale tipo di immagine dimostra quanto D. Stanojević si sia lasciato trasportare dalla propria immaginazione e dalla propria creatività nella traduzione, allontanandosi dall'originale.

Il suo narratore è molto diverso, il suo linguaggio non appartiene all'ambiente cortigiano e non è raro che l'effetto comico sia molto più basso di quello ariostesco. Narrando un grande numero di scene in prima persona, Stanojević trasforma notevolmente lo stile ariostesco, privandolo di vari riferimenti culturali. Cambia radicalmente lo stile anche nella traduzione delle strofe iniziali in cui dà del "tu" al duca Alfonso, con l'impiego di parole sempre meno formali, che appartengono al registro colloquiale: né che poco io vi dia da imputar sono che quanto io posso dar, tutto vi dono (Ariosto 2016: 36, 3). D. Stanojević scrive (1895: 1, 3): A ti ne zamjeri ako malo dadem, jer evo ti dajem baš sve što imadem / e non prendertela con me se ti do poco, perché ti do tutto quel che ho, mentre D. Angjelinović rispetta i costumi e traduce (1953: 7, 3): Da malo dajem neka me ne kore: darivam sve vam, što se dati more "che poco io vi dia non mi rimprovino, perché Le do tutto quel che posso dare". Il narratore di D. Stanojević continua a rivolgersi al duca in modo informale. È un costume ben diverso e lontano da quello ariostesco e da quello della corte dell'epoca. 
Al contrario, D. Angjelinović tiene al linguaggio sottile e sublime cercando di rispettare l'originale in questo ambito, conscio che l'eleganza rinascimentale e il linguaggio vicino al pubblico cortigiano sono onnipresenti nel poema di L. Ariosto in quanto parte inscindibile della sua ironia. D. Angjelinović cerca di rispettare e conservare questo spirito, mentre D. Stanojević continua perdendone una grande quantità, trasformando parole ed espressioni auliche in modi di dire popolari. I versi ironici nel suo poema sono quindi privati del senso (o dell'intensità) originale: quando leggiamo l'intervento del narratore (2016: 41, 22): Oh gran bontà de' cavallieri antiqui!, "attacco di una stanza nella quale l'apparente adesione al mito dell'antichità viene subito stemperata dall'ironia" (Casadei 2008: 171), D. Angjelinović traduce rispettando quasi perfettamente il senso (1953: 9, 22): Dobroto velja drevnih kavalira, mentre D. Stanojević $(1895:$ 5, 22) scrive: Volim doba starog, običaje divne!. D. Stanojević modifica un'altra volta il modo impersonale in cui è scritto l'intervento, lo scrive in in prima persona e inserisce il verbo volim/mi piacciono. I cavallieri antiqui non vengono nemmeno menzionati, diventando "tempi passati" e "costumi meravigliosi". In questo modo, si perdono di vista importanti vittime dell'ironia.

Definire il Furioso come "poema dell'ironia" (Jossa 2017: 192) significa indicare l'importanza del suo ruolo nelle tecniche narrative del Furioso. F. De Sanctis (1996: 453) descrive "l'ironia ariostesca come strumento di distanziamento dal mondo rappresentato", e come si vede, conicide con il ruolo degli interventi di L. Ariosto. L'intervento di L. Ariosto: ecco il giudicio uman come spesso erra! (Ariosto 2016: 37, 7) viene tradotto in modi diversi: D. Angjelinović (1953: 7, 7): (Gle kako ljudski sud pogriješi lako!); D. Stanojević (1895: 2, 7) O kako se ljuto mogu varat'ljudi!. Nella prima versione, i versi sono scritti tra parentesi, a differenza dell'originale, ma le parole chiave giudicio uman e erra vengono preservate; D. Stanojević trasforma invece il giudicio uman nel nome ljudi, cioè "gente", mentre il verbo erra diventa varat', un verbo che può avere più significati: la sua accezione e le diverse connotazioni non sono precisate nel contesto. Esso comprende i significati dei verbi italiani "imbrogliare", "ingannare" e "tradire". È fondamentale conservare il sintagma il giudizio umano e l'esatto significato del verbo "errare", verbo particolarmente indicativo di reminiscenze medievali ed etimologicamente collegato all'"errore" petrarchesco: sicuramente scelto da L. Ariosto anche per questi motivi, ma trattato in modo originale, senza connotazioni religiose. Questo verbo indica anche l'azione e la direzione del movimento nel poema (mostrato anche attraverso il frequente impiego delle parole "di qua, di là", di giù di su - parole chiave e spesso ignorate da D. Stanojević - nella traduzione dei versi delle ottave 13 e 31, e invece trasmesse da D. Angjelinović), giacché i personaggi si perdono nel movi- 
mento circolare, ritornando spesso nei luoghi che erano riusciti a lasciare con difficoltà. Il loro giudicio è oggetto di ironia, perché la forza principale che provoca lo svolgimento dell'azione nel poema (e nella vita, secondo L. Ariosto), procede soprattutto dall'arbitrarietà della fortuna, vista come "l'ironia del caso, ugualmente riconosciuta come ironia cosmica o ironia dell'occasione" (Hutcheon 2005: 3). [Senza] "una voce ironica, volta a proteggere, in qualche modo, il lettore dagli effetti della follia universale" (Ferretti 2017: 330) l'effetto ironico e spesso anche comico non sarebbe stato ottenuto con la stessa efficacia. "Inoltre, essendo ironico, l'ironista può essere compreso come quello che fa un "one-man show": assume il ruolo di un ingenuo e parla, scrive o si comporta come se veramente fosse il tipo di persona che potrebbe condividere il punto di vista che l'ironia intende distruggere o rovesciare" (Muecke 1986: 68).

Le capacità umane e il potere della ragione sono sottoposte al caso. Ciò viene mostrato non solo tramite il movimento circolare casuale, ma anche attraverso la psicologia dei personaggi, soprattutto attraverso la follia del protagonista, che è incapace di accettare l'impossibilità di non ottenere ciò che vuole perché il suo destino è fuori dal suo controllo, il che mostra "l'inquietudine dell' io" che "nasce dall' oscillazione del desiderio" (Praloran 2009: 180). D. Stanojević traduce il quarto verso dell'ottava 12 per strano caso uscito era di mano con la parola upusti "perse", mentre D. Angjelinović riconosce l'importanza e traduce slučajem čudnim "per uno strano caso".

D. Stanojević non traduce nemmeno i versi della strofa 26 a lungo cercasse invano ${ }^{4}$, ovviamente trascurando oppure non avendo capito l'importanza della ricerca che provoca l'azione, che si mostrerà anche come la causa principale della follia di Orlando. Questi errori sono l'esito delle incomprensioni nella prima fase del lavoro traduttivo: "dalla scorretta comprensione del contesto (del pensiero dell'autore)", "degli elementi stilistici dell'espressione linguistica: dell'ambiente, delle connotazioni ironiche o tragiche" (Levy 1982:36-37). Orlando segue Angelica "che fugge e che con la sua fuga riesce a ispirare la vita al mondo della narrazione, e si direbbe anche al mondo reale" (Milinković 2016: 44) ed è un paladino audace, ma il caso sembra burlarsi di lui mentre succede l'inaspettato: la donna amata gli fugge in tutti modi straordinari. "L'intero movimento narrativo procede dal caso, perché l'andamento razionale del meccanismo causa-conseguenza è ormai esploso e non può essere recuperato" (Jossa 2017: 191). A. Ciccarelli descrive Orlando anche in questo modo, in confronto ai personaggi pratici, come protagonista di un mondo cavalleresco destinato a fallire:

${ }^{4}$ Angjelinović $(1953: 9,26)$ invece preserva il senso e traduce: iskao dugo uzalud "cercò invano per molto tempo". 
È proprio la sua nobiltà d'animo che lo rende un personaggio ideale e poco pratico, quasi agisse fuori luogo e fuori tempo [...] come fosse un esiliato, prigioniero di modi e abitudini estranee alla sua nuova quotidianità (Ciccarelli 2017: 669).

Tradurre la situazione segnata dall'ironia cosmica senza l'uso della parola fortuna, fa perdere almeno una parte dell'ironia presente nell'originale. Questa parola, dominante nel poema, non viene resa allo stesso modo dai due traduttori: D. Angjelinović la traduce in due modi, secondo il contesto sreća "fortuna" (1953: 9, 27), o zla sreća "cattiva fortuna" $(1953: 8,10)$, mentre D. Stanojević la traduce talvolta come sudba "sorte" $(1895: 6,27)$ e altre come sreća "fortuna" $(1895: 12,57)$. Succede anche che D. Stanojević trascuri l'importanza e il ruolo di questa parola nel poema e semplicemente non la traduca. Ne mostreremo un esempio che è anche evidenza della frequente arbitrarietà della traduzione di D. Stanojević:

E come quei che non sapean se l'una o l'altra via facesse la donzella (però che senza differenzia alcuna apparia in amendue l'orma novella) si messero ad arbitrio di fortuna Rinaldo a questa, il Saracino a quella. Pel bosco Ferrau molto s'avvolse, e ritrovossi al fine onde si tolse. (Ariosto 2016: 42, 23).
Kojom cura zaždi? To nijesu znali.

Od toga im ništa nije bilo teže.

Obadva su puta razgledati stali, Al'oba tragove imali su svježe. Na misao srećnu tad oni su pali: Svak nek pravcem raznim siječe (il' reže). Mavar k mjestu starom preko novih staza Stiže, pošto dugo tamo amo baza. (Stanojević 1895: 5, 23).

In questo caso, vediamo che D. Stanojević non traduce il sintagma l'arbitrio di fortuna, che è molto importante nel rappresentare "coincidenza, caduta e curiosità" che "governano il suo mondo" (Jossa 2017: 192), ma lo trasforma in misao sretnu "un'idea felice" e così diminuisce il suo ruolo ancora una volta. Questo esempio della traduzione di un'ottava di D. Stanojević, dimostra la sua disinvoltura rispetto all'originale e il procedere liberamente: il verso od toga im ništa nije bilo teže semanticamente non trova corrispondenza nell' originale, ma è stato scritto soltanto per preservare la rima con l'uso della parola teže. L'enjambement negli ultimi due versi viene preservato, ma quello che cambia è il ritmo, dato dalla virgola che manca nel settimo verso di D. Stanojević che ne impedisce la pausa prima della parola stiže. Un'altra debolezza nella traduzione di D. Stanojević è anche l'uso dei turchismi che modifica l'immagine mentale della scena e dei personaggi ariosteschi ${ }^{5}$.

${ }^{5}$ Il turchismo presente nella traduzione "baza-basmak" è uno degli esempi dell'adattamento del poema da parte di Stanojević alla civiltà serba: il vocabolario è veramente vicino al linguaggio usato nella Serbia dell'epoca, perciò si perdono le particolarità della civiltà italiana nel poema. Tratteremo questo argomento nelle pagine successive. 
Il frequente ignorare il valore della parola "fortuna" da parte di D. Stanojević riduce l'importanza del suo ruolo nel poema: nell'ottava 44, i sintagmi fortuna crudel e fortuna ingrata vengono completamente ignorati nella sua traduzione, mentre D. Angjelinović preserva il senso e traduce srećo kruta e srećo nesmiljena (10: 44). Nell'ottava 10, presaga che quel giorno rubella dovea Fortuna alla cristiana fede è tradotto da D. Stanojević come sluteć" pogibiju hrišćanskog plemena "prevedendo la morte dei cristiani" (3: 10), senza sottolineare l'ironia cosmica rappresentata dalla Fortuna rubella, mentre Angjelinović traduce kršćansku vojsku zla sreća da bije "cattiva fortuna seguirà l'esercito cristiano" (8: 10), avvicinandosi al senso voluto nell'originale, ma non riesce a mostrare tutte le sue sfumature che producono l'ironia, siccome la sua fortuna non è rubella/ribelle, particolarmente e intenzionalmente avversa, ma solo cattiva, come casuale.

Generalmente, Angjelinović cerca di essere il più fedele possibile nella traduzione, occupandosi di trasporre l'ironia, ma come vediamo in alcuni casi sembra non aver riconosciuto l'ambiguità dell'espressione e, conseguentemente, l'immagine comica voluta da Ariosto non viene trasmessa ai suoi lettori. Così, nella strofa sessantasei si perde una parte dell'effetto comico nei seguenti versi:

Sospira e geme, non perché l'annoi che piede o braccia s'abbi rotto o mosso, ma per vergogna sola, onde a' di suoi né pria, né dopo il viso ebbe sì rosso: e più, ch'oltre al cader, sua donna poi fu che gli tolse il gran peso d'adosso. (Ariosto 2016: 53, 66)
Uzdiše, stenje, al ne što je bio

Slomio noge, uganuo ruke,

Nego jer sram mu lica nije mio,

Prije ni kaš'nje, sa toliko bruke;

Al nakon pada gospin glas mu mio

Olakša teret i u duši muke.

(Angjelinović 1953: 12, 66)

La parola "peso" ha un doppio senso, traducibile in serbo e in croato. È chiaro che, nella versione originale, il "peso" cui si riferisce L. Ariosto, non è solo un peso metaforico, ma anche peso fisico: il corpo del cavallo caduto sopra Sacripante. Il cavaliere è umiliato non solo perché Angelica è testimone della sconfitta, ma anche perché lei lo aiuta a liberarsi dal peso del cavallo. Comunque, nella traduzione, la valenza metaforica della parola "peso" anticipa il contenuto dell'ottava seguente poiché Angelica cercherà di consolarlo (ovvero togliergli il peso metaforico), incolpando l'inutile cavallo e sottolineando la fuga dell'avversario. Dunque, in questo senso, la presenza di Angelica è anche un conforto per Sacripante. D. Angjelinović, scrivendo negli ultimi due versi della strofa che la soave voce della sua donna attenuò il peso e il tormento della sua anima, riconosce solo questa valenza metaforica della parola anticipando al lettore il contenuto dell'ottava seguente. In questo modo si elimina una sosta voluta da L. Ariosto e 
l'effetto ironico della fine dell'ottava, inteso come "climax dell'azione" (Praloran 1990: 203).

La traduzione di questo verso in modo esatto (senza aggiungere la soave voce della donna, ovvero ignorando la valenza fisica dell'immagine: il momento in cui Angelica lo libera dal cavallo) è cruciale perché, nel suo doppio senso mostra ingegnosamente la tendenza di Ariosto che ama passare "da una narrazione di eventi, a una serie di sentimenti" (Praloran 1990: 217), lo fa proprio genialmente creando questa ambiguità. Il traduttore ovviamente non percependo l'immagine inventata da L. Ariosto, ne trova la spiegazione solo nell'ottava seguente (capisce in quella parte che il peso nel cuore è diminuito grazie alle parole di consolazione) e lo attribuisce direttamente ai versi dell'ottava precedente, per darne un'immagine più chiara ai lettori. E anche se questa fosse stata l'unica valenza della parola "peso", l'anticipazione non sarebbe giustificata: D. Angjelinović, non avendo capito l'ambiguità e tradotto il senso, ha eseguito un altro errore comune: anticipando non ha rispettato l'unità strofica dell'originale.

D. Stanojević invece rispetta in questo caso l'unità strofica, riconosce solo la valenza fisica della parola, ignorandone il significato metaforico e traduce questi ultimi versi sostituendo la parola peso con la parola gamba:

\section{[...] Stidi se, ljuti se na sudbinu strogu \\ jer je mor'o gledat Anđeliku mladu, kako mu izvlači ispod konja nogu [...] $(1895: 14,6)$}

In questo caso, è evidente che entrambi i traduttori non riescono a rendere il doppio senso né la sottile ironia di L. Ariosto. Nella versione di D. Stanojević l'ironia e l'ambiguità vengono ridotte a effetto comico, a una buffoneria. La complessità e le differenti sfumature dell'ironia verbale sono le più difficili da interpretare ed effettivamente da trasporre in un'altra lingua. Sebbene spesso si confondano come effetto durante la lettura del Furioso, visto che "l'umorismo sembra essere una componente importante dell'ironia situazionale e dell'atteggiamento ironico" (Kreuz 2020), esiste una chiara linea di divisione fra l'ironia e l'umorismo che possono essere anche contrapposti: "l'umorismo discende, [mentre] l'ironia è l'adozione di un punto di vista "sopra" un certo contesto, permettendoci di vederlo "dal sù"” (Colebrook 2005: 133). Lo stesso succede con la traduzione degli ultimi versi della settima ottava: Il savio imperator che estinguer vuolse un grave incendio (37: 7). La parola "incendio" ha una doppia valenza metaforica: la prima, riconosciuta e tradotta dai due traduttori, e il riferimento al disaccordo e l'eventuale battaglia fra i paladini, mentre la seconda, quella trascurata o non capita dai traduttori e il riferimento al d'amoroso desio l'animo caldo (37: 8), come L. Ariosto scriverà già nell'ottava seguente. L. Ariosto riprenderà questa metafora anche nell'ultima ottava del primo canto 
precisandola nel sintagma amoroso incendio (57: 81). Quando D. Stanojević percepisce l'incendio come neslogu i buru "disaccordo e turbamento" (2:7) e D. Angjelinović come razdor u vojsci "un disaccordo nell'esercito" (7: 7), si perde l'ironia creata dall' ambiguità dell'espressione ariostesca. L. Ariosto utilizza questa metafora nell'opera nel senso parodico della parola, al pari di Lorenzo de Medici che scrive nella sua Nencia da Barberino: ardo d'amore e conviemmi cantare ${ }^{6}$. M. Praloran riconosce la particolare connotazione parodica del linguaggio legato al "desiderio amoroso" di Petrarca e anche del mondo cavalleresco:

\begin{abstract}
"C'è certamente anche l'universo cortese, quello del desiderio amoroso. Lì certamente le due linee si incrociano, ma i cavalieri ariosteschi non hanno la stessa complessità psicologica, la stessa autenticità nell'espressione di sé che hanno i loro antenati, i cavalieri arturiani. Per questo, in bocca loro il discorso "cortese" non può non apparire un po' ironico [...] Anche da qui nasce l'ironia, o la parodia - per certi aspetti uno schermo, una sospensione del pathos - da questa non coincidenza." (Praloran 2009: 179).
\end{abstract}

L'Angelica di Ariosto non vuole che si abbia di lei sì falsa opinione, mentre quella di Stanojević proclama semplicemente che "si è imbrogliati pensando che non sia vergine" varaš se misleći da nisam nevina (1895: 11, 52). Il protagonista ariostesco proclama: Corrò la fresca e matutina rosa, [...] so ben ch'a donna non si può far cosa che più soave e più piacevol sia [...], tardando, stagion perder potria (51: 58), D. Angjelinović: jutarnju, svježu ružu ću ubrati [...] znam dobro ženi ne može se dati milije stvari, ni više voljene [...] (12: 58), D. Stanojević: Sad ubraću ružu, punu vlage rosne [...] ništa nije ženi milije i draže, no kad se muško tijela joj kosne (12: 58). La sensualità e l'ambiguità sono tradotte da D. Stanojević in immagini crude, dirette, in cui "un uomo mette le mani sopra il corpo di una donna", parole non adatte al linguaggio e alla cultura cortesi, e si perdono l'ironia, la delicatezza e la metafora della rosa. J. Levy proclama che: "[...] la dissimulazione carraterizza ugualmente la moderna costruzione dell'opera quanto gli enunciati diretti" (148).

L'immagine rappresentata da D. Stanojević è esplicita e esagerata, sembra un assalto fisico alla donna che, come continua il traduttore, ako se brani, ona samo laže "se si difende, non lo pensa veramente" (12: 58). Questa ottava, piena di ambiguità e riferimenti a motivi classici e artistici, L. Ariosto finisce con il verso ch'io non adombri e incarni il mio disegno, nel senso di "delinei" e "realizzi". Nel commentario di questa edizione del Furioso a cura di Davide Puccini, questi termini sono spiegati nel seguente modo: "[...] sono tratti dalla pittura (ombreggiare, dare il color carne), ma è

\footnotetext{
${ }^{6} \mathrm{~L}$. Medici invece mette in parodia un protagonista tipico dalla poesia pastorale.
} 
probabile, soprattutto per il secondo, un doppio senso malizioso. Del resto, incarnare è usato nel significato di "congiungersi carnalmente" dall'Aretino e dal Caro" (2016: 58). I due traduttori non trovando equivalenti si allontanano dall'originale, in direzioni diverse. D. Stanojević traduce: ispunit ću želju sasvijem po planu "soddisferò il mio desiderio come pensavo" (12: 58), e D. Angjelinović preserva la simbologia della rosa, dai versi precedenti, riutilizzandola in: neće, spriječit me, da to ne poberem cvijeće "non mi impedirà di cogliere quei fiori" (12: 58), comunque rimanendo più vicino al linguaggio e all'immagine ariostesca.

\section{IL CRONOTOPO SERBO NELLA TRADUZIONE DI STANOJEVIĆ}

Come abbiamo cominciato a mostrare in precedenza, D. Stanojević si allontana dal linguaggio della tradizione letteraria italiana e nella sua traduzione si perdono i motivi della poesia petrarchesca e rinascimentale. Lo stesso succede nella strofa quarantadue, dove si perde almeno la metà dei motivi, in cui L. Ariosto utilizza le metafore dominanti nella società cortigiana rinascimentale e nella descrizione si riferisce a Poliziano e Catullo, paragonando come di consueto una donna e una rosa cui tutta la natura si inchina per renderle omaggio:

La verginella è simile alla rosa, che in bel giardin su la nativa spina mentre sola e sicura si riposa, né gregge né pastor se le avicina; l'aura soave e l'alba rugiadosa, l'acqua, la terra al suo favor s'inchina: gioveni vaghi e donne inamorate amano averne e seni e tempie ornate. (Ariosto 2016:47, 42):
Djevica je kao ta rumena rosa, što u vrtu leška na svojemu žbunu. Mladić rado ružu za klobukom nosa, cura u kosici ili na zubunu; Slavopoj se njojzi od slavlja i kosa Za mirise njene donosi u kljunu Njenijem imenom dragi dragu krsti, Vrt je čuva da je stado ne obrsti. (Stanojević 1895: 9, 42)

L'immagine creata da D. Stanojević nel quinto, sesto e settimo verso di questa strofa si discosta enormemente dall'originale. La natura che rende omaggio alla rosa non appare nella traduzione, dove al suo posto si trova "una canzone solenne per celebrare i suoi profumi portata dal merlo nel suo becco" e "col suo nome l'amato battezza la sua amata", mentre il senso degli ultimi due versi è anticipato al terzo e quarto verso e il senso del quarto verso è trasferito all'ultimo.

Nell' ottava precedentemente citata, si nota che le parole klobuk e zubun sono aggiunte da D. Stanojević: nell'originale non vengono menzionati né "il cappello" né "la camicia". Comunque, questi esempi non servono solo a 
sottolineare la libertà smodata della traduzione di D. Stanojević, ma soprattutto per rilevare un'altra caratteristica della sua traduzione: l'uso dei turchismi. Queste due parole hanno una loro specificità, e in italiano non potrebbero essere tradotte semplicemente come "cappello" e "camicia" poiché sono specificamente legate al costume del luogo e dell'epoca in cui ha vissuto D. Stanojević. Così, per precisare il significato di zubun dovremmo aggiungere che si tratta di una camicia particolare, composta da tre pezzi e con tante decorazioni. Senza dubbio, questa camicia non è immaginabile dai lettori di L. Ariosto. Con le stesse conseguenze, D. Stanojević nel suo poema usa anche la parola mejdan (un altro turchismo, 1895: 13, 61) come traduzione di "battaglia" o "campo di battaglia". Benché l'autore non cambi i nomi dei personaggi in nomi serbi, per lui Orlando, paladino gagliardo diventa Rolando, delija ${ }^{7}(1895: 3,12)$ e possiede altre caratteristiche peculiari per la società dei lettori di D. Stanojević.

Mi sembra che non ci sia un popolo sulla terra che degrada le parole così come fanno gli italiani. Loro pronunciano Norfolk come Nortfozia, Richmond - Ritmonda, Hampton - Antona, Northumberland - Norbelanda [...] Roland è il francese principe Marco. In qual modo si pronuncia la radice di questa parola, non lo chiederò agli italiani, ma ai francesi, e io le aggiungerò la desinenza serba, come mi sembra conveniente (Stanojević 1895: 277).

Seguendo la stessa logica e considerando l'effetto melodico delle traduzioni dei nomi nel suo paese, gli altri personaggi che compaiono nella sua traduzione sono: Ferag (Ferraù), Sakripan (Sacripante), Anđelika (Angelica), Rinaldo (Rinaldo) e Bajardo (Baiardo). Angjelinović si mantiene ancora più vicino all'originale e traduce i nomi dei personaggi: Orlando, Ferrau, Sakripant, Anđelika, Rinaldo, Bajard. Si riscontra però un errore nella sua traduzione quando a un certo punto l'autore traduce Orlando invece di Rinaldo, cambiando drasticamente il contesto: dato che segue una lunga descrizione del duello, il lettore, logicamente, non capisce chi sono $\mathrm{i}$ veri protagonisti di tutta una serie di ottave. Il suo errore riguarda i seguenti versi: trasse la spada, e minacciando corse dove poco di lui temea Rinaldo (Ariosto 2016: 40, 16) tradotti in Angjelinović (1953: 8, 16): K'Orlandu jurnu on $i$ tu su se srela s mačem u ruci ova dva junaka.

D. Stanojević evoca l'ambiente serbo utilizzando metafore e modi di dire propri del suo Paese. In questo modo, le unità artistiche (i realia e il contesto) sono lontane dall'originale. Quindi, il lettore non vede la "realtà immaginata" (Levy 1982: 39) del poeta. Al contrario, Stanojević utilizza "le

7 Turchismo: un membro della speciale cavalleria ottomana della fine del '400 e dell'inizio del '500. 
soluzioni locali" (Levy 1982: 44) quando invece potrebbe usare "la lingua neutrale" necessaria solo se il valore stilistico dell'elemento è intraducibile.

Tra i numerosi esempi, citeremo la traduzione dei versi ariosteschi appartenenti all'ottava sessantaquattro: tal si levò il pagano, vengono tradotti: "così fece anche il nostro" muja (Stanojević 1895: 14, 64). Talvolta le metafore tradotte vengono aggiunte liberamente solo per conservare l'ottava rima o per ottenere l'effetto comico (anche quando Ariosto non lo fa!) e sono quasi tutte legate allo spazio quotidiano pieno di metafore che includono vari tipi di animali. Così, l'uomo innamorato è "più stupido di un bue" gluplji od vola (Stanojević 1895: 12, 56), Sacripante smonta dal cavallo apparendo "più feroce di un serpente" izgledaše jetkiji od zmije (Stanojević 1895: 9, 39) e minaccia di "schiacciare Rinaldo come una mosca" ja ću ovoga smeljat ka muhu (1895: 17, 80).

Nella sua versione le metafore sono frutto della sua pura invenzione e D. Stanojević utilizza la maggior parte di queste narrando di Angelica. Lei viene spesso comparata a "un uccello" ptičica $(1895: 12,57)$, a "una zebra leggera" kao laka zebra $(1895: 4,17)$ a "il nostro micetto" naša mala mica (1895: 8, 37), a "una preda" plijen (1895: 12, 57), e una volta anche a "una femmina spaventata" prestravljena ženka $(1895: 7,33)$, banalizzando così l'eleganza e la scelta minuziosa delle parole di L. Ariosto. La discrezione sembra mancare nelle metafore modificate o addirittura inventate nella traduzione. La scelta delle parole da parte di D. Stanojević è sempre esplicita, concreta, e in questo modo si perde il garbo e lo stile dell'originale, spostando le avventure ariostesche in un altro contesto. D. Stanojević non presta attenzione alle figure legate a questo ambito, così come non si cura di tradurre bene il seguente sintagma: Un cavalliero [...] d'aspetto fiero (Ariosto 2016: 42, 25) viene tradotto da lui semplicemente come strašan čovjek "un uomo fiero" (Stanojević 1895: 6, 25), mentre D. Angjelinović (1953: 9, 25) scrive vitez ponosna pogleda "un cavaliere dallo sguardo fiero". Tramite queste negligenze, si perde tanto della traduzione e dell'immagine che il lettore si crea mentalmente. Nell'ottava ventotto, Stanojević ignora l'importanza e il livello formale della parola valore nel codice cavalleresco, amata e usata da Ariosto, e fa una perifrasi "col tuo valore" (implica: se sei capace e onorevole) scrivendo nijesi li strina "se non sei una zia" (1895: $6,28)$, un altro modo di dire che appartiene al registro colloquiale di una realtà di cui fa parte lo stesso Stanojević, più che a una realtà lontana, di tempi passati e costumi sublimi in cui Ariosto situa il suo poema.

${ }^{8}$ Nome personale, generalmente utilizzato nel popolo per designare l'uomo comune, molto frequente in questa parte del mondo. 


\section{CONCLUSIONE}

Al fine di identificare le particolarità e i diversi modi in cui è possibile tradurre il pensiero ariostesco, in questo lavoro ci siamo concentrati sull'esempio del primo canto e abbiamo cercato di presentare i metodi traduttivi di D. Stanojević e D. Angelinović mediante un confronto comparatistico, analizzando i procedimenti con cui sono stati trattati gli elementi essenziali che costituiscono l'espressione e lo stile ariosteschi: gli interventi del narratore, l'ironia e le espressioni ambigue.

Abbiamo rilevato che la traduzione di D. Stanojević è molto arbitraria: la scelta del metodo traduttivo deriva dalle necessità culturali dell'epoca. Senza rispettare completamente il senso, il livello del linguaggio, lo stile e le tecniche narrative, nell'impiego del principio di accettabilità e non cessando di aggiungere elementi propri nella traduzione, l'Orlando furioso viene trasformato in un altro poema cavalleresco. Per questo motivo abbiamo trovato particolarmente necessario dedicare un capitolo alla creazione del "cronotopo serbo" nella sua traduzione. Non si può trascurare l'apprezzabilità e la bellezza di questo poema, ma è pur sempre evidente che la grazia dell'originale Orlando furioso è ben altra e appartiene a un'altra civiltà. Le intenzioni di D. Stanojević spiegate nelle lettere inviate alla Cooperativa letteraria serba rendono palesi le sue priorità: presentare il poema ariostesco ai serbi, tenendo però conto "dell'orecchio serbo". Il traduttore si propone di arricchire la letteratura serba e perde di vista il Furioso. Tenendo conto solo dei destinatari e talvolta mancando di percepire appieno la realtà immaginata di L. Ariosto, la modifica e crea il cronotopo serbo.

Al contrario, D. Angjelinović si preoccupa devotamente di trasferire il senso, di riproporre le tecniche narrative quanto più possibile, di riprodurre il linguaggio e l'ambiente cortigiano di L. Ariosto. Non si può negare che abbia raggiunto lo scopo, pur avendo mostrato anche i lati meno riusciti della sua traduzione: gli errori involontari nella traduzione e i rari (ma importanti!) casi in cui sembra non aver colto totalmente l'ironia di L. Ariosto. Rispetto a D. Stanojević, D. Angjelinović ha un doppio vantaggio: la sensibilità culturale dei suoi lettori nei confronti della società italiana e in ugual misura le esperienze e le conoscenze personali legate al territorio italiano e alla letteratura italiana. Per tale ragione, D. Angjelinović, attraverso il principio dell'adeguatezza, presenta in modo abbastanza verosimile una traduzione e non una nuova versione del Furioso. 


\section{BIBLIOGRAFIA}

Angjelinović, D. (1953). Bijesni Orlando. Zagreb: Zora.

Ariosto, L. (2016). Orlando furioso. Roma: Newton Compton editori s.r.l. Avirović, Lj. (2006). La divina traduzione. Tradurre in croato dall'italiano. Trieste: EUT-Edizioni.

Casadei, A. (2008). Nuove prospettive su Ariosto e sul "Furioso". Italianistica: Rivista Di Letteratura Italiana 37, 3, 167-192. Testo disponibile a JSTOR, www.jstor.org/stable/23937911. (11.1.2021.).

Ciccarelli, A. (2017). Nota sulla follia di Orlando in Ariosto. Italica 94, 4, 665-684. Testo disponibile a JSTOR, www.jstor.org/stable/44983628. (11.1.2021.).

Colebrook, C. (2005). Irony. London and New York: Taylor \& Francis e-Library.

De Sanctis, F. (1996). Storia della letteratura italiana, a cura di N. Gallo. Torino: Einaudi Gallimard.

Eco, U. (2013). Dire quasi la stessa cosa. Milano: Bompiani.

Ferretti, F. (2017). Stilistica e genere cavalleresco. A proposito di due recenti lavori di Maria Cristina Cabani. Lettere Italiane 69, 2, 328-337. Testo disponibile a JSTOR, www.jstor.org/stable/26562957 (11.1.2021.).

Hutcheon, L. (2005). Irony's edge. London and New York: Taylor \& Francis e-Library.

Jankelevitch, V. (1997). L'ironia. Genova: Il nuovo melangolo.

Jossa, S. (2017). Ironia. In A. Izzo (a cura di), Lessico critico dell'Orlando Furioso (pp. 177-197). Roma: Carocci.

Kadić, A. (1962). The Croatian Renaissance. Slavic Review 21, 1, 65-88. Testo disponibile a JSTOR, www.jstor.org/stable/3000544. Accessed 8 Mar. 2021.

Kreuz, R. (2020). Irony and sarcasm. Cambridge: The MIT Press Essential Knowledge Series.

Levy, J. (1982). Umjetnost prevođenja. Sarajevo: Svjetlost.

Malinar, S. (2002). Italiano e croato a contatto sulla costa orientale dell'Adriatico. Dai primi secoli all'Ottocento (I). Studia Romanica et Anglica Zagrabiensia 47-48, 283-309.

Milinković, S. (2016). S ženom valja kao s fortunom. Ljubavni i diskurs o ženi u italijanskoj književnosti XIV-XVI veka. Književna istorija $2,29-48$.

Morini, M. (2015). La traduzione: teorie, strumenti, pratiche. Milano: Sironi.

Muecke, D. C. (1986). Irony and the Ironic. New York: Methuen \& Co.

Musić, S. (1975). Dragiše Stanojević prevod Bijesnog Rolanda. Beograd: Institut za književnost i umetnost. 
Praloran, M. (1990). Meraviglioso artificio. Tecniche narrative e rappresentative nell'Orlando Innamorato. Lucca: Maria Pacini Fazzi editore.

Praloran, M. (2009). Le lingue del racconto. Studi su Boiardo e Ariosto. Roma: Bulzoni.

Rivoletti, C. (2014). Ariosto e l'ironia della finzione - La ricezione letteraria dell'Orlando furioso in Francia, Germania e Italia. Venezia: Marsilio.

Stanojević, D. (1895). Bijesni Rolando. Biograd: Srpska književna zadruga.

Steiner, G. (1998). After Babel-Aspects of language and translation. USA: Oxford University Press.

Toury, G. (2012). Descriptive translation studies and beyond. Amsterdam: John Benjamin's B.V.

Zogović, M. (1972). Stavovi Dragiše Stanojevića o prevođenju Bijesnog Rolanda. Zbornik Matice srpske za književnost i jezik 20 (2), 386-408.

\section{IRONY AND NARRATORIAL INTERVENTIONS IN THE TRANSLATIONS OF THE FIRST CANTO OF ORLANDO FURIOSO \\ IN SERBO(CROATIAN)}

\section{Summary}

In this article, we attempt to analyze some elements of the translations of the first canto of Orlando furioso, with the intention of comparing the results obtained by different translation principles present in Dragiša Stanojević's and Danko Angjelinović's translations. Because the two translators were oriented toward different translation priorities and norms, the narratorial interventions, irony, and some ambiguous words and expressions in relation to Ariosto's specific historical and cultural context appear very differently in the two metatexts. The primary objective of our analysis is to demonstrate, through a comparative approach, the results of two different translation principles that have created an Orlando adapted to the Serbian chronotope and another, a true ambassador of Italian culture and literature in the territory of former Yugoslavia, both in relation to the translators' historical and cultural circumstances and to the aspirations of the epochs in which the translations have been made.

Keywords: translation principles, interventions, chronotope, court, irony, ambiguity. 